\title{
Nasogastric administration of lenvatinib solution in a mechanically ventilated patient with rapidly growing anaplastic thyroid cancer
}

\author{
Fumiaki Kawano1, Tadato Yonekawa2, Hideki Yamaguchi², Nobuhiro Shibata ${ }^{3}$, \\ Kousei Tashiro1, Makoto Ikenoue', Shun Munakata', Kazuhiro Higuchi', \\ Hiroyuki Tanaka ${ }^{4}$, Yuichiro Sato4, Ayumu Hosokawa ${ }^{3}$, Shinsuke Takeno', \\ Kunihide Nakamura ${ }^{1}$ and Atsushi Nanashima ${ }^{1}$
}

Departments of ${ }^{1}$ Surgery, ${ }^{2}$ Neurology, Respirology, Endocrinology and Metabolism, ${ }^{3}$ Clinical Oncology, and ${ }^{4}$ Diagnostic Pathology, Faculty of Medicine, University of Miyazaki, Miyazaki, Japan

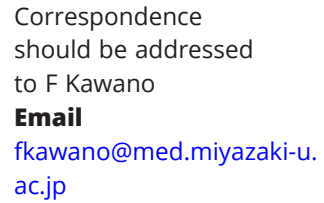

\section{Summary}

A 54-year-old woman was referred to our hospital with a cervical tumor. CT revealed a cervical tumor extending to the upper mediastinum, tracheal deviation and tumor infiltration in the cervical vessels. She was followed-up because no diagnosis of malignancy was made by cytology. However, 2 months later, a CT scan showed enlargement of the tumor and tracheal stenosis, and a surgical biopsy was performed and she was diagnosed with anaplastic thyroid cancer (ATC). The tracheal tube with tracheal stenosis could not be removed due to the rapid growth of the tumor, necessitating management by mechanical ventilation. Due to the difficulty of surgical resection, she was treated with lenvatinib. A lenvatinib solution was made and administered via a nasogastric tube. After lenvatinib treatment, the tumor volume decreased and the tracheal stenosis improved. The tracheal tube was removed and oral intake became possible. She was discharged and received ambulatory lenvatinib therapy. The tumor was significantly reduced in size, but gradually grew and was exposed through the cervical wound 6 months later. Esophageal perforation occurred 10 months after the start of treatment. Lenvatinib was re-administered via a nasogastric tube. Eleven months later, the patient died of massive bleeding from the exposed cervical tumor. Patients with advanced ATC may require management with mechanical ventilation for airway stenosis or with a nasogastric tube for esophageal stenosis and perforation. We experienced a case in which lenvatinib was safely administered via a nasogastric tube while performing mechanical ventilation.

\section{Learning points:}

- An anaplastic thyroid cancer patient under mechanical ventilator management was treated with lenvatinib via a nasogastric tube.

- The lenvatinib solution can easily be prepared and administered via a nasogastric tube.

- The lenvatinib solution was effective for a patient with difficulty in oral intake.

- Lenvatinib could also improve the prognosis of an anaplastic thyroid cancer patient with severe airway and esophageal trouble. 


\section{Background}

Anaplastic thyroid cancer (ATC) is rare and is the most aggressive malignancy. ATC grows rapidly and easily invades the surrounding organs, such as the trachea and esophagus (1). It may, therefore, occasionally be necessary to manage cases of the airway or esophageal trouble (2). The standardized treatment for ATC is a multimodal approach and the combination of surgery and external radiation with chemotherapy; however, the effects of several tyrosine kinase inhibitors (TKIs) have been confirmed in recent years (3). Lenvatinib is an oral, multi-targeted, tyrosine kinase inhibitor of vascular endothelial growth factor receptors 1, 2 and 3; fibroblast growth factor receptors 1-4; platelet-derived growth factor receptor alpha; and the RET and KIT signaling pathways (4). Lenvatinib has been used to treat many ATC patients and the efficacy and safety have been reported. However, there have been few reports on the efficacy and safety in patients under mechanical ventilator management or that have described the administration of lenvatinib via a nasogastric tube. We herein report a case of ATC with stenosis of the trachea and the esophagus, in which lenvatinib was administered via a nasogastric tube to a patient under mechanical ventilator management.

\section{Case presentation}

A 54-year-old woman presented to a primary care hospital with a history of shoulder pain, dysphagia, and hoarseness. CT revealed a large tumor from the cervical area to the upper mediastinum. Fine-needle aspiration biopsy of the tumor was performed but no malignancy was diagnosed. She was, therefore, referred to our institution. Surgical biopsy was performed, but the pathological examination did not suggest malignancy. Since the patient's symptoms were mild and she was not diagnosed with any malignancy, she was followed-up conservatively. Two months later, her dysphagia worsened and she developed dyspnea on exertion. A CT scan revealed that the tumor had grown significantly. She was, therefore, urgently admitted to our hospital.

\section{Investigation}

A physical examination revealed the following: body temperature, $37.5^{\circ} \mathrm{C}$; blood pressure, $115 / 61 \mathrm{mmHg}$; heart rate, 101 b.p.m.; and respiratory rate, 10 breaths/ min. A hard mass was palpable on the left clavicle and stridor was heard on auscultation of the cervical field.
A laboratory analysis revealed an elevated WBC count $\left(13800 / \mathrm{mm}^{3}\right)$ and C-reactive protein level $(6.7 \mathrm{mg} / \mathrm{dL})$. A thyroid function test demonstrated a TSH level of $1.50 \mu \mathrm{U} / \mathrm{mL}$ and an FT4 level of $1.06 \mathrm{ng} / \mathrm{dL}$. Her serum thyroglobulin level was $2.02 \mathrm{ng} / \mathrm{mL}$. No tumor marker abnormalities were observed. A CT scan showed that the tumor had reached the aortic arch and the origin of the left common carotid artery and the subclavian artery were also more strongly involved (Fig. 1). Furthermore, the trachea and esophagus were severely displaced on the right and remarkable stenosis of the trachea was recognized. There were no findings suggesting distant metastases. The diagnosis of the tumor was necessary in order to plan her treatment. Furthermore, tracheal intubation had to be considered because her dyspnea rapidly worsened after hospitalization. We performed an incisional biopsy of the cervical tumor under general anesthesia. A collar sharped incision was made and a portion of the tumor was exposed. The tumor was not in contact with the thyroid and was not a primary tumor of the thyroid. An intraoperative frozen section analysis yielded a diagnosis of squamous cell cancer; thus, the wound was closed and the operation was completed. A histological examination revealed nest-like proliferation of epithelioid tumor cells, with enlarged hyperchromatic nuclei and notable nucleoli in fibrocollagenous tissue (Fig. 2). Immunohistochemistry was positive for PAX8, TTF-1, and 34bE2 and negative for thyroglobulin, CD5 and CD117 (Fig. 2). The proliferation index Ki-67 (MIB-1) was $60 \%$. The pathological findings were compatible with
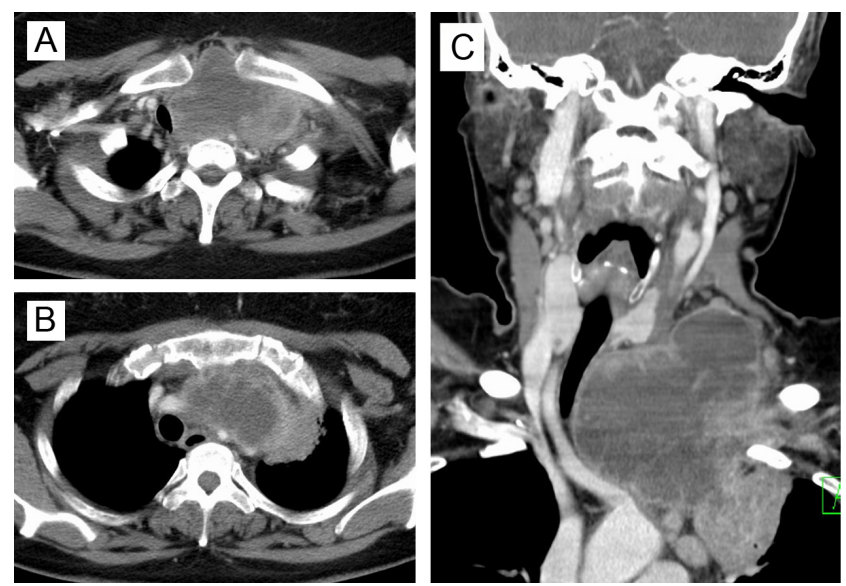

Figure 1

Contrast-enhanced CT imaging performed when the patient was referred to our department. (A) The cervical tumor was extending to the upper mediastinum and displacing the trachea and esophagus. (B) The tumor infiltrated the left common carotid artery, the subclavian artery, and the left pleura. (C) The tumor reached the aortic arch and was not continuous with the thyroid. 

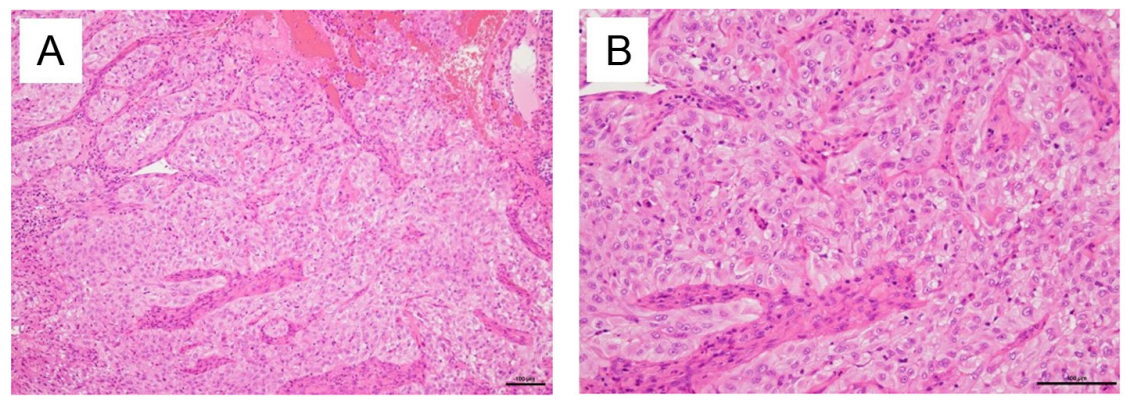

\section{D}
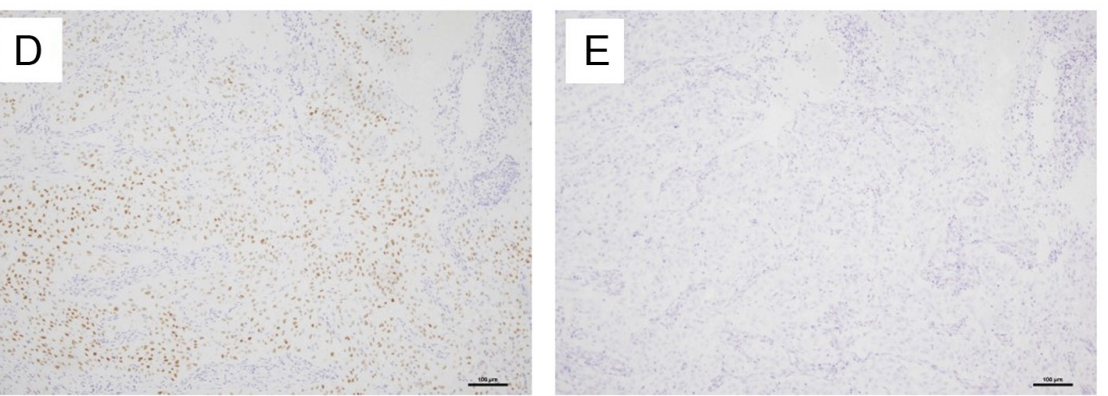
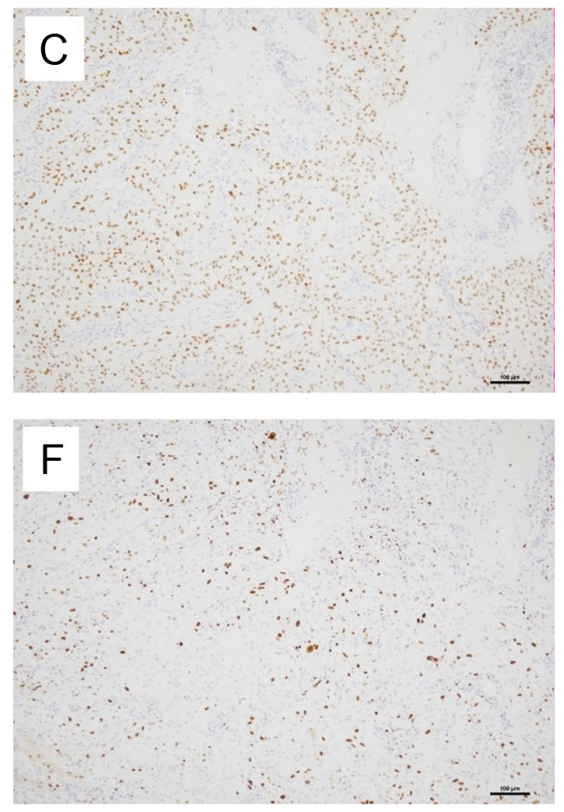

Figure 2

Histopathological findings of the cervical tumor. (A) Hematoxylin-eosin staining (magnification, $\times 20$ ). (B) Hematoxylin-eosin staining (magnification, $\times 100$ ). (C) PAX8 staining (magnification, $\times 20$ ). (D) TTF-1 staining (magnification, $\times 20)$. (E) Thyroglobulin staining (magnification, $\times 20)$. (F) Ki-67 staining (magnification, $\times 20)$.

anaplastic thyroid cancer with a squamous cell cancer component.

\section{Treatment}

The patient received medical management in an intensive care unit postoperatively and it was not possible to remove the tracheal tube. CT revealed severe cervical edema and tracheal stenosis (Fig. 3A). Although the risk of perforation of the trachea and arteries was suggested, we decided to use lenvatinib as a treatment strategy for ATC. Lenvatinib therapy was started and administered at a dose of $10 \mathrm{mg} /$ day. Because oral intake was not possible, it was injected into the patient's stomach via a nasogastric tube. The lenvatinib solution was prepared by mixing $20 \mathrm{~mL}$ of $55^{\circ} \mathrm{C}$ saline solution and a $10 \mathrm{mg}$ drug capsule, waiting for $10 \mathrm{~min}$, and then stirring; only the solution was collected. In this way, lenvatinib solution was easily administered via a nasogastric tube. At 10 days from the start of therapy, CT revealed a reduction in the tumor volume and tracheal compression (Fig. 3B). The patient's tracheal stenosis improved after the start of therapy and her tracheal tube was removed. After extubation, the oral administration of lenvatinib became possible. After treatment with lenvatinib, the cervical incisional wound was spontaneously dissected and necrotic tumor tissue was discharged from inside.

\section{Outcome and follow-up}

Although the dose of lenvatinib had to be changed due to adverse drug events of proteinuria (grade 3) and hypertension (grade 3), she received ambulatory treatment. Three months later, the tumor was significantly reduced and the solid component had almost disappeared (Fig. 4A). A cavity in the mediastinum was identified from the cervical wound. Six months later, a CT scan revealed that the tumor was growing and the tumor was exposed through the cervical wound (Fig. 4B). Ten months later, oral lenvatinib capsule was expelled from the cervical wound. Because esophageal perforation was confirmed on $\mathrm{CT}$, the administration of lenvatinib was discontinued (Fig. 5A). However, remarkable tumor growth was confirmed after the cessation of lenvatinib, and lenvatinib therapy was restarted via a nasogastric tube (Fig. 5B). Thereafter, the tumor growth was slow; however, her clinical condition rapidly deteriorated. At 11 months from the start of therapy, the patient died of massive bleeding from an exposed cervical tumor. In autopsy findings, the tumor had extensively invaded surrounding organs and the left common carotid artery wall was ruptured. There was no 

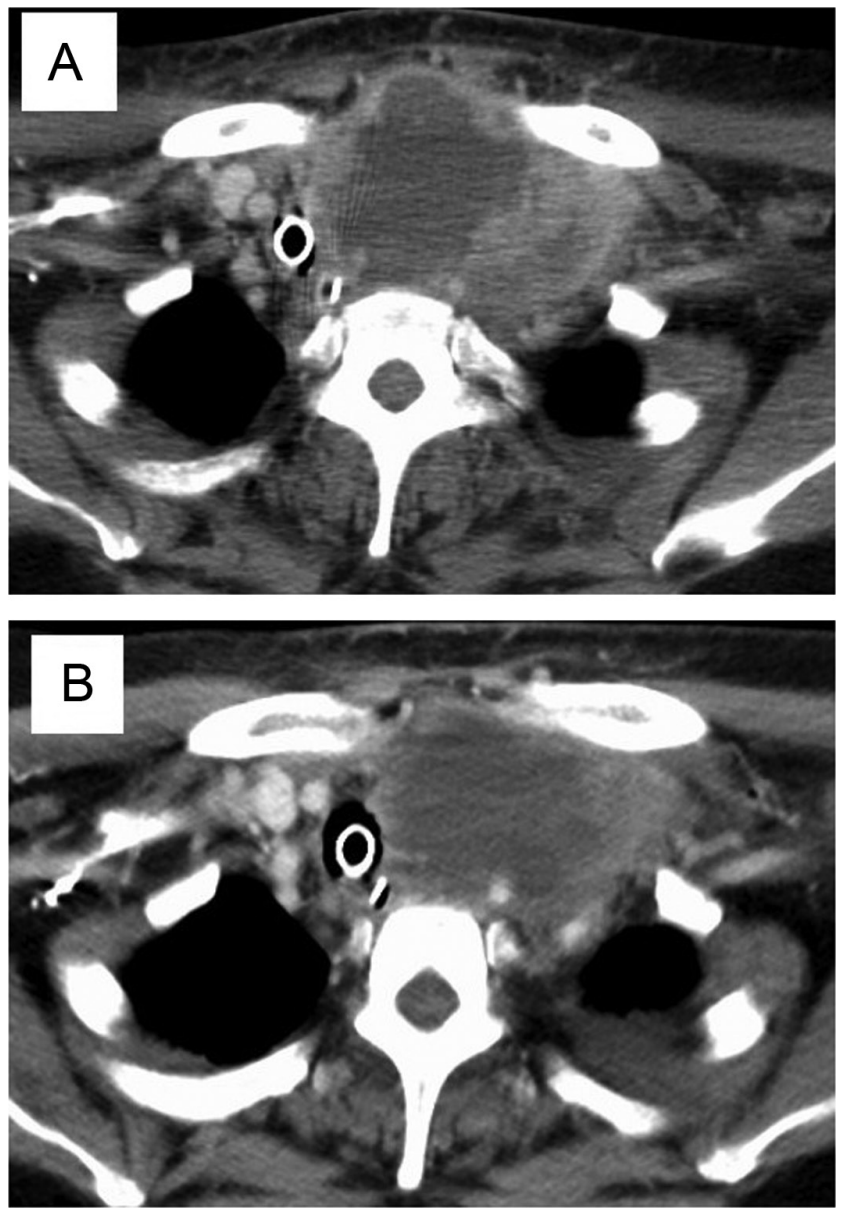

\section{Figure 3}

$\mathrm{CT}$ imaging before and after lenvatinib treatment. (A) The cervical tumor before the administration of lenvatinib. (B) Ten days after administration of lenvatinib, a reduction of the tumor volume and tracheal compression was observed.

evidence of distant metastasis, except for a small focal lesion in the adrenal gland.

\section{Discussion}

The effects of several tyrosine kinase inhibitors (TKIs) in the treatment of advanced or radioiodine refractory thyroid cancer have been confirmed in recent years. The TKIs sorafenib and lenvatinib, including cabozantinib, vandetinib, axitinib, and sunitinib, have also been shown to be effective in the management of advanced differentiated thyroid cancer (3). Lenvatinib is a multitargeted, tyrosine kinase inhibitor of the vascular endothelial growth factor receptors (VEGFRs) 1, 2 and 3; fibroblast growth factor receptors 1-4; platelet-derived growth factor receptor alpha; and the RET and KIT signaling pathways (4). It has also been reported that ATC patients treated with lenvatinib presented an acceptable
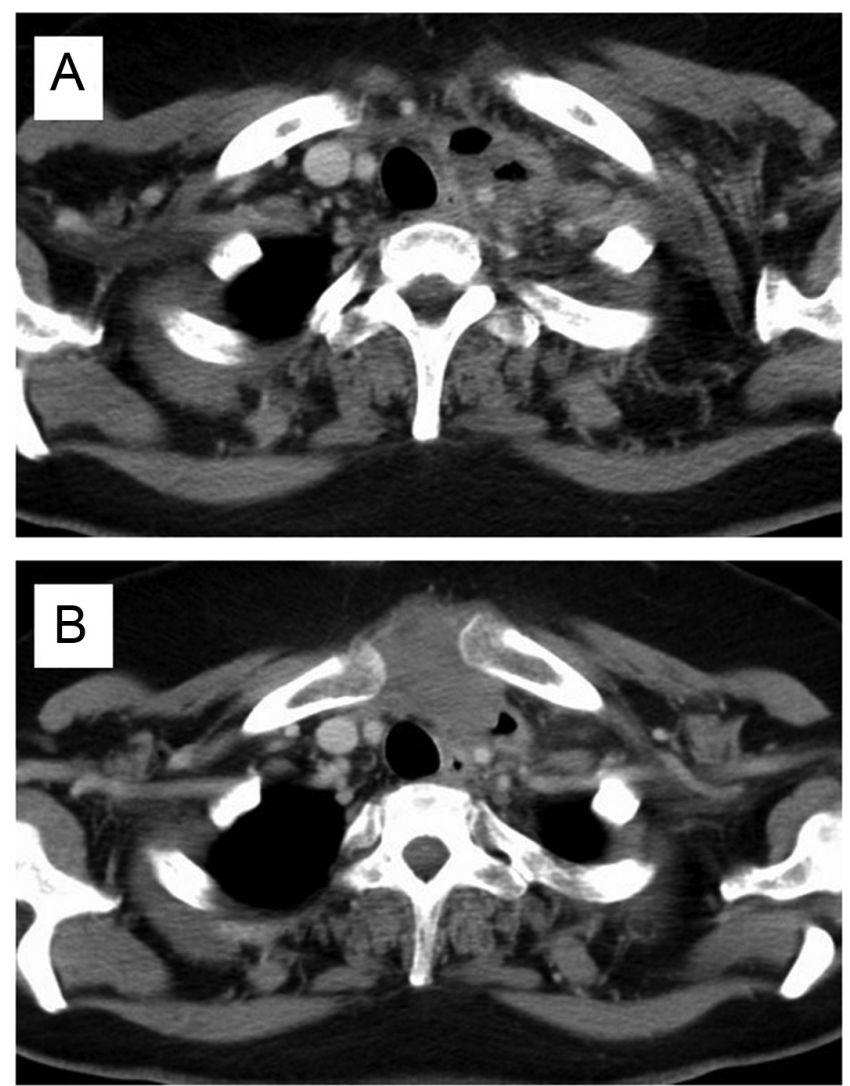

Figure 4

CT imaging at 3 and 6 months after lenvatinib treatment. (A) Three months after administration, the tumor was significantly reduced and the solid component had almost disappeared. (B) Six months later, it was confirmed that the tumor was growing.

response rate and disease control rate $(4,5)$. It could be a breakthrough treatment for cases for which there was no effective treatment previously. Although the efficacy and safety of lenvatinib for ATC patients have been largely accepted, it has been recognized that lenvatinib is associated with a high rate of treatment-related adverse events, including hypertension and proteinuria. Further caution is required in relation to wound-healing complications or perforation of the adjacent invading organs in patients undergoing VEGFR inhibitor treatment. Tumor invasion of vital structures, such as the trachea, esophagus and carotid artery, might be fatal due to the antioncotic effect of lenvatinib $(4,6)$. Thus, we must consider the indications for the initiation of lenvatinib treatment.

ATC represents the most aggressive and deadly thyroid malignancy (1). ATC is most commonly found in the context of a rapidly growing thyroid mass, along with vocal cord paralysis, dyspnea, dysphagia, and neck pain. The trachea, esophagus, recurrent laryngeal 

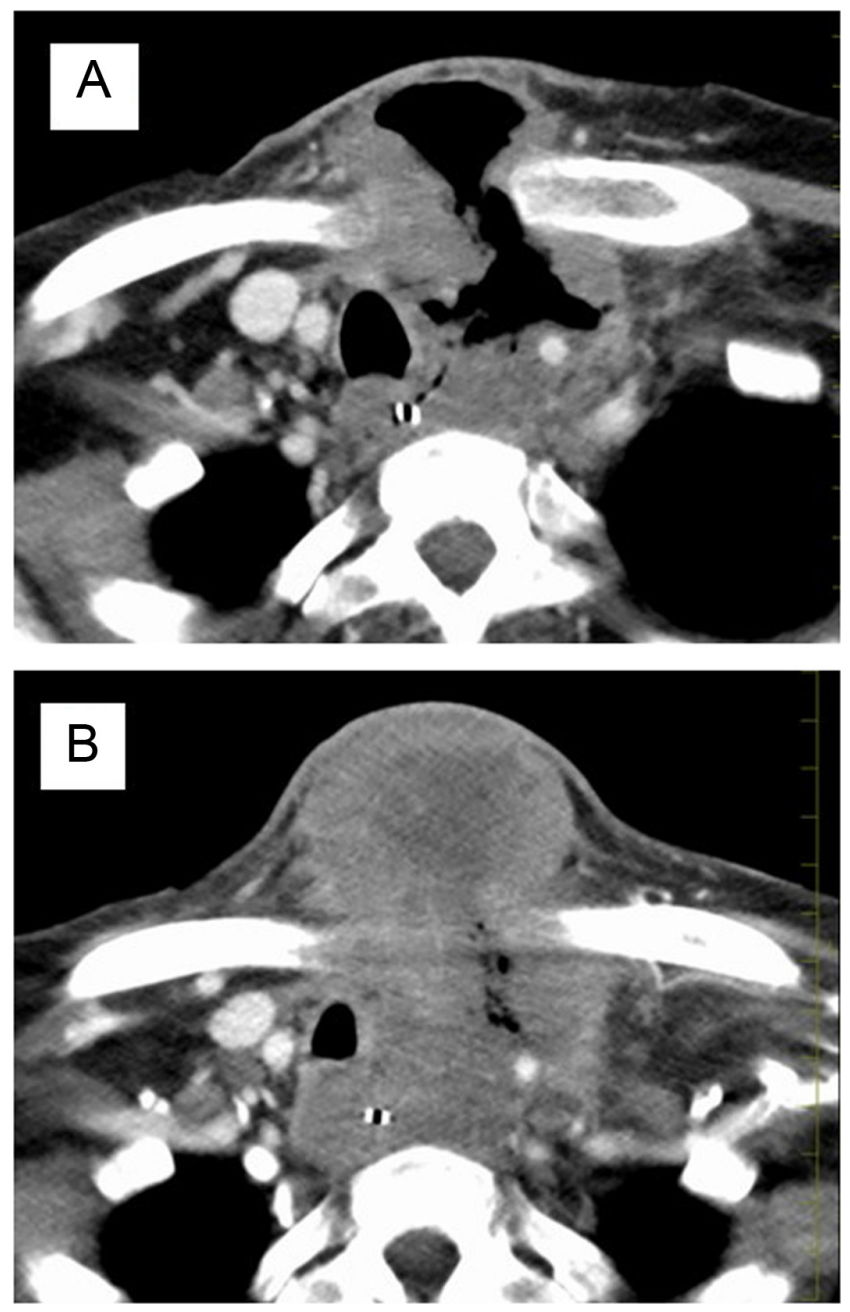

Figure 5

CT imaging before and 1 week after the discontinuation of lenvatinib treatment (A) At 3 months after administration, esophageal perforation appeared. (B) At 1 week after the discontinuation of lenvatinib treatment, remarkable tumor growth was confirmed.

nerve and cervical vessels around the thyroid are easily infiltrated, with the patient presenting multiple progressive symptoms. Therefore, most patients have difficulty in radical operation and must be combined with external radiation, chemotherapy, and targeted therapies. The clinical effects of taxanes, doxorubicin, and platins have also been reported, and in some cases, long-term survival has been achieved in combination with surgery or radiation therapy. The local control ability of external radiation was also recognized. However, the survival rate was not improved significantly in the last 30 years. Furthermore, it is necessary to deal with various troubles that occur during the process. One of the most common and difficult problems in the management of ATC is securing the airway. Approximately $20-30 \%$ of ATC patients present with acute airway problems, which may require airway management, such as tracheal intubation or tracheotomy (2). Esophageal stenosis associated with tumor infiltration and compression is also a problem. Enteral injection, such as nasogastric tube or gastrostomy, may also be necessary in cases in which oral intake is difficult.

The efficacy and safety of chemotherapy for patients with mechanical ventilation have not been established. There have been several reports on chemotherapy for patients under mechanical ventilation. Koba et al. (7) reported a case of mediastinal small cell lung cancer in which the patient was managed with mechanical ventilation due to tracheal stenosis. Egyed et al. (8) reported a case of Burkitt lymphoma in which laparotomy was required for abdominal compartment syndrome. These patients were successfully treated with chemotherapy and finally extubated. There has been a case report of the administration of a TKI to a thyroid cancer patient under mechanical ventilation due to respiratory failure as a result of multiple pulmonary metastases (9). This patient was treated with sorafenib and the tracheal tube was temporarily extubated. We consider chemotherapy under mechanical ventilation to be acceptable if therapeutic effects can be expected. However, although this management is acceptable for ATC patients, tracheostomy should be avoided for airway control. Tracheostomy is often difficult because the trachea cannot be exposed to the invading tumor as tumor growth through the incision site may become uncontrollable (2). Furthermore, the administration of lenvatinib can lead to tracheostomy problems due to impaired wound healing. In recent years, various tracheal stents have become clinically available and expected to have clinical utility.

Lenvatinib is an oral tyrosine kinase inhibitor. It is administered as a powder inside a capsule. For patients with esophageal stenosis and dysphagia, it can be administered using a nasogastric tube. Eleonora et al. (10) reported a case in which lenvatinib administered via a nasogastric tube was effective in the treatment of a differentiated thyroid cancer patient with esophageal stenosis. Lenvatinib is sparingly soluble in aqueous buffers. It should first be dissolved in dimethyl sulfoxide and then diluted with the aqueous buffer of choice (https://www. caymanchem.com/product/19375/lenvatinib, Accessed 2 April 2020). We prepared a lenvatinib solution by mixing $20 \mathrm{~mL}$ of $55^{\circ} \mathrm{C}$ saline solution and a $10 \mathrm{mg}$ drug capsule. The solution was easily collected in a syringe for injection. Furthermore, the $\mathrm{pH}$ value of the lenvatinib solution was in the range of $7.3-7.5$, and there was no 
restriction on the material of the tube when injecting. In our cases, lenvatinib was successfully administered by these methods. Decapsulation of lenvatinib and administration via nasogastric tube is an acceptable and effective method. However, gastrostomy and enterostomy should be avoided if lenvatinib is administered, as there is a risk of unexpected trouble, such as peritonitis, due to impaired wound healing.

In the present case, the patient was diagnosed with ATC; however, extubation was difficult due to tracheal deviation and stenosis. The patient was managed with a mechanical ventilator without tracheotomy or stenting due to concerns about healing failure in association with TKI therapy. Lenvatinib therapy was effective: it reduced the tumor volume and improved the tracheal deviation and stenosis. As a result, the tracheal tube could be removed and oral intake became possible. Lenvatinib was administered via a nasogastric tube, even in the terminal stage when the patient's esophagus and tumor/ skin were in communication and she could not take the medicine. To our knowledge, this is the first report on the administration of lenvatinib via a nasogastric tube to an ATC patient under mechanical ventilation. Considering the clinical characteristics of ATC, lenvatinib treatment may be necessary for patients with tracheal or esophageal problems. This report may be helpful for patients who require ventilator management or tube administration. This therapeutic method may be adapted to many cases in the future.

\section{Declaration of interest}

The authors declare that there is no conflict of interest that could be perceived as prejudicing the impartiality of the research reported.

\section{Funding}

This research did not receive any specific grant from any funding agency in the public, commercial, or not-for-profit sector.

\section{Patient consent}

Written informed consent for publication of the clinical details and images was obtained from the patient's sister.

\section{Author contribution statement}

F Kawano, H Yamaguchi, K Nakamura, and A Nanashima wrote the case report. $\mathrm{H}$ Tanaka and $Y$ Sato prepared and commented on the pathological findings. T Yonekawa, N Shibata, K Tashiro, M Ikenoue, S Munakata, K Higuchi, A Hosokawa, and S Takeno contributed to the clinical management of this patient.

\section{References}

1 Ain KB \& Valentino J. Anaplastic thyroid cancer and thyroid lymphoma. In Surgery of the Thyroid and Parathyroid Glands, pp 242-249. Ed GW Randolph. Philadelphia: Elsevier, 2012.

2 Shaha AR, Ferlito A, Owen RP, Silver CE, Rodrigo JP, Haigentz M, Mendenhall WM, Rinaldo A \& Smallridge RC. Airway issues in anaplastic thyroid carcinoma. European Archives of Oto-RhinoLaryngology 2013270 2579-2583. (https://doi.org/10.1007/s00405013-2556-3)

3 Yu ST, Ge JN, Luo JY, Wei ZG, Sun BH \& Lei ST. Treatment-related adverse effects with TKIs in patients with advanced or radioiodine refractory differentiated thyroid carcinoma: a systematic review and meta-analysis. Cancer Management and Research 201911 1525-1532. (https://doi.org/10.2147/CMAR.S191499)

4 Tahara M, Kiyota N, Yamazaki T, Chayahara N, Nakano K, Inagaki L, Toda K, Enokida T, Minami H, Imamura Y, et al. Lenvatinib for anaplastic thyroid cancer. Frontiers in Oncology 20177 25. (https:// doi.org/10.3389/fonc.2017.00025)

5 Iwasaki H, Yamazaki H, Takasaki H, Suganuma N, Nakayama H, Toda S \& Masudo K. Lenvatinib as a novel treatment for anaplastic thyroid cancer. A retrospective study. Oncology Letters 201816 7271-7277. (https://doi.org/10.3892/ol.2018.9553)

6 Obata K, Sugitani I, Ebina A, Sugiura Y, Toda K, Takahashi S \& Kawabata K. Common carotid artery rupture during treatment with lenvatinib for anaplastic thyroid cancer. International Cancer Conference Journal 20165 197-201. (https://doi.org/10.1007/s13691016-0257-7)

7 Koba T, Hirata H, Kijima T, Naito Y, Hamaguchi M, Suga Y, Kuroyama M, Koyama S, Iwahori K, Takimoto T, et al. A case of mediastinal small cell lung cancer in a patient receiving mechanical ventilation who was successfully treated with chemotherapy and finally extubated. Gan To Kagaku Ryoho 201845 1335-1337 (in Japanese).

8 Egyed E, Heiss MM, Wappler F \& Sakka SG. Successful treatment of abdominal compartment syndrome with chemotherapy in a patient with a newly diagnosed Burkitt lymphoma. Journal of Critical Care 201951 26-28. (https://doi.org/10.1016/j.jcrc.2019.01.021)

9 Iyer P, Mayer JL \& Ewig JM. Response to sorafenib in a pediatric patient with papillary thyroid carcinoma with diffuse nodular pulmonary disease requiring mechanical ventilation. Thyroid 2014 24 169-174. (https://doi.org/10.1089/thy.2012.0468)

10 Eleonora M, David V, Nicola V, Pierpaolo F, Francesca O, Liborio T, Paola V, Alessandro R, Gabriele M, Paolo V, et al. Lenvatinib administered via nasogastric tube in poorly differentiated thyroid cancer. Case Reports in Endocrinology 20192019 1-4. (https://doi. org/10.1155/2019/6831237)

Received in final form 15 June 2020 Accepted 13 July 2020 